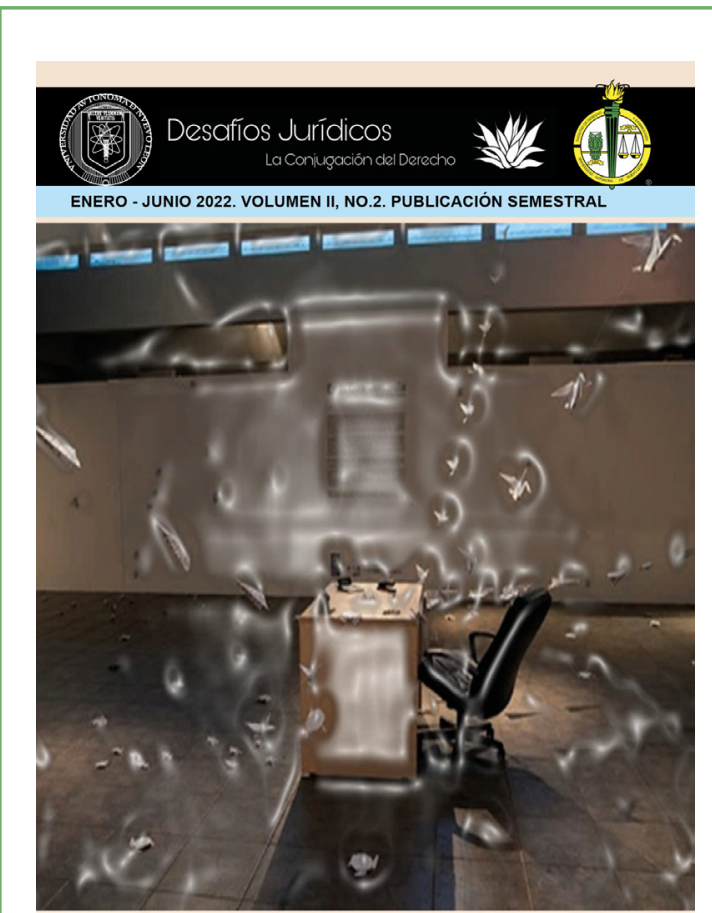

Detalles sobre la publicación, incluyendo instrucciones para autores e información para los usuarios en: https://desafiosjuridicos.uanl.mx/ index.php/ds

Ivonne Janeth Ixba Santiago \& Brenda Judith Sauceda Villeda (Facultad de Derecho y Criminología, Universidad Autónoma de Nuevo León)

La capacidad jurídica del adulto mayor maduro. pp. 90-111 Fecha de publicación en línea: 31 de enero del 2022.

Publicado en Desafíos Jurídicos La Conjugación del Derecho. Todos los derechos reservados. Permisos y comentarios, por favor escribir al correo electrónico: desafios.juridicos@uanl.mx

Desafíos Jurídicos La Conjugación del Derecho. Revista de temas contemporáneos sobre derecho, y sus conexiones en la vida cotidiana, Volumen 2, No. 2, enero-junio de 2022, es una publicación semestral de la Universidad Autónoma de Nuevo León, a través de la Facultad de Derecho y Criminología, editada en la Ciudad Universitaria, N.L, México. Con dirección en Cd. Universitaria, Av. De los Rectores s/n, San Nicolás de los Garza, N.L. C.P. 66451, Página electrónica de la revista: http://desafiosjridicos.uanl.mx

Editora en jefe: Dra. Amalia Guillén Gaytán Reserva de Derechos al Uso Exclusivo del Título Volumen 2, No. 2, enero-junio de 2022, ISSN: en trámite ante el Instituto Nacional del Derecho de Autor. Responsable de la última actualización de este número: Mtro. Juan Alonso Martínez Arrieta.

Desafíos Jurídicos La Conjugación del Derecho aborda temas contemporáneos sobre derecho, y sus conexiones en la vida cotidiana, tiene como propósito constituirse en un foro de discusión académica que aborda la compleja, contradictoria y multicausal relación entre el derecho y la vida social. Desafíos Jurídicos se inscribe en el debate académico nacional e internacional en el ámbito de Derecho y su giro especial en las ciencias sociales e invita al análisis de diversas prácticas sociales y formas de organización y acción política desde una perspectiva multidisciplinaria que ponga énfasis en la defensa de los derechos y su aplicación. Los textos publicados incorporan métodos y problemas tratados desde el derecho, la sociología, la ciencia política, la economía, los estudios urbanos, la geografía, los estudios culturales, la antropología, la literatura y el feminismo, entre otros. Las opiniones expresadas por los autores no reflejan la postura del comité editorial.

Queda estrictamente prohibida la reproducción total o parcial de los contenidos e imágenes de la publicación sin previa autorización expresa de la revista.

DIRECTORIO INSTITUCIONAL

RECTOR: DR. SANTOS GUZMÁN LÓPEZ

SECRETARIO GENERAL: DR. JUAN PAURA GARCIA

DIRECTOR DE LA FACULTAD DE DERECHO Y CRIMINOLOGIA: MTRO. OSCAR P. LUGO SERRATO

REVISTA DESAFÍOS JURÍDICOS

DIRECTORA: Dra. Amalia Guillén Gaytán

CORDINADOR: Dr. Mario Alberto García Martínez

CORDINADOR DEL NÚMERO: Mtro. Juan Alonso Martínez Arrieta

ASISTENTE EDITORIAL: Mtra. Angélica Rubí Rodríguez Aguirre

ADMINISTRACIÓN DEL SITIO WEB: M.A. Daniel Vázquez Azamar

EDICIÓN TEXTUAL Y CORRECCIÓN DE ESTILO: Rosa María Elizondo Martínez

PINTURA DE LA PORTADA: M.A. Daniel Vázquez Azamar "En la otra ventanilla" (C 2022 


\section{La capacidad jurídica del adulto mayor maduro}

The legal capacity of the mature older adult

Fecha de publicación en línea: 31 de enero del 2022

Por: Ivonne Janeth Ixba Santiago* \& Brenda Judith Sauceda Villeda**

**https://orcid.org/0000-0003-3614-7875

Universidad Autónoma de Nuevo León

Resumen. El siguiente artículo pretende hacer una aproximación del bioderecho a la compleja realidad del mundo con base a la capacidad legal del adulto mayor maduro en su etapa de envejecimiento. La principal intención de este texto es analizar la diferencia entre las clasificaciones que dentro de la etapa de la vejez se localizan los adultos mayores la cual ha afectado de manera especial a la población antes referida, centrándonos en definir que el envejecimiento derivado por los cambios fisiológicos no ocurre de la misma forma en cada sujeto, y los prejuicios legales como sociales han posicionado a cada adulto mayor en una situación de vulnerabilidad. El objeto principal de estudio es analizar la capacidad legal a través del proceso del envejecimiento, mediante los prejuicios legales y sociales que se encuentran en las políticas públicas en el ámbito médico, lo que nos permitirá hacer comparaciones con las leyes que se han decretado en el Estado de Nuevo León. La intención es crear una visión mediante la cual se distingan las diferentes clasificaciones del adulto mayor como respuesta al cambio por parte del envejecimiento, que partiendo de la teoría existente sobre que los cambios fisiológicos no son impedimentos para que el adulto mayor maduro se encuentre sin aptitud de ejercer su voluntad, debido a que no causan una incapacidad de ejercicio. El presente estudio se realiza desde una metodología descriptiva con análisis de contenido documental en diversas fuentes del derecho internacional, derecho interno de Nuevo León y revistas científicas.

Palabras clave: Adulto Mayor Maduro, capacidad jurídica, bioderecho, envejecimiento, principio de autonomía y autorrealización, principio de dignidad humana. 
Abstract. The following article intends to make an approach of biolaw to the complex reality of the world based on the legal capacity of the mature elderly in their aging stage. The main intention of this text is to analyze the difference between the classifications that within the stage of old age are located the older adults which has affected in a special way the population referred to above, focusing on defining that aging derived by physiological changes does not occur in the same way in each subject, and legal and social prejudices have positioned each older adult in a situation of vulnerability. The main object of the study is to analyze legal capacity through the aging process, by means of the legal and social prejudices found in public policies in the medical field, which will allow us to make comparisons with the laws that have been enacted in the State of Nuevo León. The intention is to create a vision through which the different classifications of the older adult are distinguished as a response to change on the part of aging, based on the existing theory that physiological changes are not impediments for the mature older adult to be unable to exercise his or her will, since they do not cause an inability to exercise. This study es based on a descriptive methodology with documentary content analysis in various sources of international law, domestic law of Nuevo León and scientific journals.

Key words: Mature Older Adult, legal capacity, biolaw, aging, principle of autonomy, principle of human dignity.

\section{INTRODUCCIÓN}

En el presente estudio se utiliza una metodología descriptiva, realizando un análisis de contenido documental tomando de base como referencias principales Liliam Flores, Carla Villarreal y Pablo Simón-Lorda; asimismo, se consideraron diversas fuentes del derecho internacional como la Declaración Universal de los Derechos Humanos y la Ley de los Derechos de las Personas Adultas Mayores, y del derecho mexicano, particularmente del Estado de Nuevo León como la Ley General de Salud del Estado de Nuevo León y la Ley de los Derechos de las Personas Adultas Mayores en el Estado de Nuevo León.

Históricamente, las personas que se encontraban con una discapacidad intelectual 0 mental fueron considerados como individuos incapaces de tomar sus propias decisiones: Reconocido así en el Código de Napoleón en su título XI, sobre la mayoría de edad y de los mayores que están protegidos por la ley, indica que "el mayor de edad que se encuentre en un estado habitual de imbecilidad, de demencia o de furor debe ser sujeto a interdicción, aun cuando ese estado presente intervalos lucidos" ${ }^{1}$; fue así que en el siglo XIV,

Hernández, Mónica, "El Concepto de Discapacidad: De la enfermedad al enfoque del Derecho". Revista CES Derecho, vol.6, no.2, noviembre 2015. Recuperado el 22 de enero de 2022 de: http://www.scielo.org.co/pdf/cesd/v6n2/v6n2a04.pdf\#: : text=En\%20\%C3\%A9pocas\%20hist\%C3\%B3ricas $\% 20$ la\%20discapacidad $\% 20$ fue $\% 20$ objeto $\% 20$ de,sus $\% 20$ facultades $\% 20$ intelectualesse $\% 20$ denominaban $\% 20 \%$ E2\% $80 \%$ 9Cmente\%20captus. \%E2\%80\%9D\%20\%28Mu\%C3\%- 
los nacidos con deficiencias ya sea físicas, sensoriales o mentales, como la sordera, la ceguera, la parálisis, la cuadriplejía, entre otros, eran confinados a grandes encierros, en los que eran exhibidos los fines de semana a manera de espectáculo circense o de gran zoológico, para que las familias se divirtieran un poco o bien, manejando la conciencia social, rectificaran los actos cometidos en el pasado, por considerar a estos monstruos o fenómenos como la más grande señal de un castigo enviado por Dios ${ }^{2}$.

Establecido de esta manera respecto a los discapacitados mentales se generó un rechazo de parte de la sociedad al limitarlos en elegir o expresar su voluntad en su vida cotidiana, inclusive ignorando su existencia como persona y dignidad humana. Sin embargo, el Derecho ha creado diversas figuras con la finalidad de proteger a las personas que padecen de una incapacidad natural y legal, o sólo una de ellas. Al respecto el artículo 450 inciso II del Código Civil de Nuevo León nos menciona:

"Tienen incapacidad natural y legal: Los mayores de edad, con incapacidad o discapacidad originada por enfermedades o deficiencias persistentes de carácter físico, psicológico o sensorial que les impida gobernarse por sí mismos o no

B1oz\%2C\%202010\%29

2 Santofimio, German "De la anomalía a la discapacidad, una larga historia de exclusión social: De la muerte, al destierro y el repudio, a la inclusión educativa". Revista Inclusión y Desarrollo, vol. 3, no. 1, 2016. Recuperado el 22 de enero de 2022 de: http://biblioteca.uniminuto.edu/ojs/index.php/IYD/ issue/archive puedan manifestar su voluntad por algún medio". ${ }^{3}$

De este modo, dicho precepto refiere que se considera a la persona incapaz materia de estudio, en dos situaciones diferentes: la primera que por una causa de enfermedad que ocasione en su inteligencia perturbación alguna que le impida ejercer su voluntad con los demás aun cuando pudiera desaparecer al recuperar su salud como intervalos de lucides; y la segunda que sea una enfermedad irreversible como la persona que no podrá nunca más recuperar la salud mental que le permita comprender, o que por algún estado de discapacidad no pueda gobernarse por sí mismo, al obligarse o manifestar su voluntad por algún medio.

En el camino del desarrollo como seres humanos en la vida se tienen etapas que vivir; iniciando con la niñez, pasando por la adolescencia, la adultez, y terminando en la vejez, siendo esta última etapa en la que el ser humano al igual que en la niñez necesita una protección debido al deterioro físico que se produce por el tiempo e inclusive debido al deterioro mental, es decir, en adultos mayores se presenta la demencia.

Siguiendo esta línea el envejecimiento no implica enfermedad, dependencia y falta de productividad necesariamente, sin embargo, conforme se van añadiendo años a la vida

3 H. Congreso del Estado de Nuevo León (2021), "Código Civil para el Estado de Nuevo León", Recuperado el 26 de septiembre de 2021 de: http://www.hcnl.gob.mx/trabajo_legislativo/leyes/codigos/codigo_civil_para_el_estado_de_ nuevo_leon/ 
también se van incorporando en las personas mayores diversas modificaciones fruto del proceso natural de envejecimiento que conllevan cambios en las estructuras internas y externas. ${ }^{4}$ De esta manera los cambios se presentan en físicos, fisiológicos, psicológicos, cognitivos, psicomotores y socioafectivos: Por lo cual, el deterioro mental en el estado de vejez se puede presentar como demencia la es definida por la Organización Mundial de la Salud (OMS) como:

"Un síndrome generalmente de naturaleza crónica o progresiva caracterizado por el deterioro de la función cognitiva, la cual afecta a la memoria, el pensamiento, la orientación, la comprensión, el cálculo, la capacidad de aprendizaje, el lenguaje y el juicio que suele ir acompañado, y en ocasiones es precedido, por el deterioro del control emocional, el comportamiento social o la motivación, pero la conciencia no se ve afectada". ${ }^{5}$

Conforme a datos del Censo 2020 proporcionado por el Instituto Nacional de Estadística y Geografía $^{6}$ (INEGI) indica que en el país hay

4 González Jerónimo y Fuente Raquel, “Desarrollo humano en la vejez: un envejecimiento optimo desde los cuatro componentes del ser humano". INFAD Revista de Psicología, vol. 7, núm. 2, 2014. Recuperado el 22 de enero 2022 de: https:// www.redalyc.org/pdf/3498/349851791013.pdf.

5 Organización Mundial de la Salud, Temas de Salud, Centro de Prensa "Demencia". Recuperado el 9 de octubre de 2021 de: https://www.who.int/es/news-room/fact-sheets/detail/dementia

6 Instituto Nacional de Estadística y Geografía, Censo de población y vivienda 2020, Comunicado de prensa num.24/21 del 25 de enero de 2021. Recuperado el 9 de octubre de 2021
20838108 personas con alguna limitación, discapacidad o problema o condición mental, mismas que representan $16.5 \%$ de la población, de estas, 1590583 (1.3\%) tiene algún problema o condición mental.

Es importante destacar que, en esta última etapa al generalizar la ancianidad con todos los adultos mayores, se cae en el error de creer que todos tienen las mismas capacidades de goce y ejercicio, pero tanto "la vejez" como "adulto mayor (AM)" tienen diferentes clasificaciones, por tanto, no todos se encuentran en la misma situación.

En los últimos años, el $\mid \mathrm{NEGI}^{7}$ señala que la población total del Estado de Nuevo León, del 2010 a 2020, aumentó de 4,653,458 a $5,784,442$ tantos de personas y que, respecto a la población adulta mayor de 60 años o más, esta cifra pasó de 407,278 (8,8\%) a 654,050 (11.3\%). Por lo anterior, se puede establecer que Nuevo León ha sufrido un crecimiento demográfico del $3 \%$ sobre la población de objeto de estudio, tal como se puede observar en el transcurso de los últimos diez años, que ha pasado de una población de jóvenes a una población adulta, debido al incremento de personas de 60 años y más.

Por tanto, en la entidad hay cada vez un aumento de adultos mayores, que requieren de

de: https://www.inegi.org.mx/contenidos/saladeprensa/boletines/2021/EstSociodemo/ResultCenso2020_Nal.pdf

7 Instituto Nacional de Estadística y Geografía, Censo de población y vivienda 2020, "Presentación de Resultados de Nuevo León”. Recuperado el 9 de octubre de 2021 de: https://www.inegi.org.mx/contenidos/programas/ccpv/2020/ doc/cpv2020_pres_res_nl.pdf 
una protección a sus derechos humanos, a recibir los satisfactores necesarios para su atención integral, incluyendo su acceso preferente a los servicios médicos y a recibir capacitación y orientación respecto a su salud, nutrición, higiene y todos aquellos aspectos que favorezcan su cuidado personal ${ }^{8}$, entre los cuales podemos hacer mención importante a los principios de autonomía y autorrealización, así como al principio de la dignidad humana. Vinculado a esto, se establece en el artículo $3^{\circ}$ de la Ley de Derechos de las Personas Adultas Mayores en el Estado de Nuevo León que son "aquellas que tienen sesenta años o más de edad y que se encuentren domiciliadas $o$ de paso en el Estado de Nuevo León". ${ }^{9}$ Por tal razón, jurídicamente el AM en la ley estatal es reconocido como sujeto al cual se debe garantizar el ejercicio de sus derechos, tales como a recibir una atención médica y a contar con los servicios de salud conforme a las disposiciones jurídicas que sean aplicables.

De este modo, el principio de la autonomía y autorrealización es fundamental en el ámbito médico, al reconocer al individuo como sujeto de derecho a tomar sus propias elecciones y a mantener diferentes criterios, mediante el cual el profesional del ámbito médico le otorga la

8 Comisión Nacional de los Derechos Humanos, "Los Derechos Humanos de las Personas Mayores". Recuperado el 9 de octubre de 2021 de: https://www.cndh.org.mx/sites/all/doc/ cartillas/2015-2016/27-DH-Adultos-Mayores.pdf.

9 Congreso del Estado de Nuevo León, "Ley de los Derechos de las Personas Adultas Mayores en el Estado de Nuevo León", Recuperado el 27 de septiembre de 2021 de: http:// www.hcnl.gob.mx/trabajo_legislativo/leyes/leyes/ley_de_los_ derechos_de_las_personas_adultas_mayores_en_el_estado_de_nuevo_leon/ información respecto a su salud al asegurarse que el individuo de este estudio comprende lo que se le comunica, y por lo tanto se respeta su voluntad y participación.

En la actualidad hay diversas formas con las cuales se pueden referir a los adultos mayores, entre las cuales se mencionan personas de la tercera edad, ancianos, adultos de edad avanzada, senectud, entre otros, sin embargo, sin importar la palabra que se utilice, durante esta etapa las personas necesitan de mayor protección, debido a la situación en la que se encuentran se convierte en seres más vulnerables. En tal sentido, como lo define la Comisión de Atención a Grupos Vulnerables que, por su condición de edad, sexo, estado civil y origen étnico, se encuentran en condición de riesgo que les impide incorporarse al desarrollo y acceder a mejores condiciones de bienestar. ${ }^{10}$

\section{CONCEPTO DE ENVEJECIMIENTO: BREVE EVOLUCIÓN Y CONNOTACIONES}

Si bien, en el ámbito social al utilizar las palabras "personas adultas mayores", suele asimilarse con envejecimiento, al definirlas como personas frágiles, que se enferman con facilidad o no pueden realizar acciones $u$ actos de ningún tipo, personas vulnerables que no puede protegerse por sí mismos, a pesar de que el envejecimiento es una palabra diferente a adultos mayor, no similares, pero si existe una

10 Flores, Liliam, "Grupos Vulnerables", Recuperado el 26 de septiembre de 2021 de: http://archivos.diputados.gob.mx/ Centros_Estudio/Cesop/Eje_tematico_old_14062011/9_gvulnerables_archivos/G_vulnerables/d_gvulnerables.htm 
asociación entre ellos. ${ }^{11}$ Por lo tanto, el factor social influye de gran importancia debido a que puede ser causada por una persona o por todo un grupo, la cual desencadena factores que impidan que el individuo pueda desarrollar su dignidad humana.

Se puede apreciar en la definición de la OMS en el informe mundial sobre el envejecimiento y la salud publicado en el 2015 "son consideradas personas de la edad avanzada las que tengan de 60 a 74 años, considerada vieja o ancianas a las que tienen 75 a 90 años, y se les denomina viejos o grandes si sobrepasan los 90 años". ${ }^{12}$ Por lo tanto, establece que se le llamará en forma indistinta persona de la tercera edad a todo individuo mayor de 60 años.

El envejecimiento activo ha sido definido por la OMS ${ }^{13}$ como la optimización de oportunidades de salud, participación y seguridad con el fin de mejorar la calidad de vida del envejecimiento. Por lo que el envejecimiento, puede ser desde un aspecto positivo, en el que el AM puede desarrollarse libremente en la sociedad

11 Alvarado, A; Salazar (2014), "Análisis del concepto de envejecimiento", Revista Scielo, 2, Recuperado el 15 de septiembre de 2021 de: https://scielo.isciii.es/scielo.php?script=sci_arttext\&pid=S1134-928X2014000200002

12 Organización Mundial de las Naciones Unidas, "Informe mundial sobre el envejecimiento y la salud", https://apps.who. int/iris/bitstream/handle/10665/186466/9789240694873_spa. pdf? sequence $=1$

13 Varela Pinedo, Luis F., "Salud y calidad de vida en el adulto mayor", Revista Peruana de Medicina Experimental y Salud Pública, vol.33, no.2, abril-junio 2016. Recuperado el 15 de septiembre de 2021 de: http://www.scielo.org.pe/scielo. php?script=sci_arttext\&pid=S1726-46342016000200001 con las capacidades que cuenta, sin más limitantes que su edad, para seguir con su vida de manera normal, porque sin importar la palabra que se utilice, durante esta etapa las personas adultas necesitan de mayor protección por parte del Estado con la creación de mecanismos que los ayuden a conseguirlos.

A lo largo de las etapas de la vida que atraviesa todo ser humano, pasa por una serie de cambios físicos y biológicos. Por lo tanto, cada persona envejece de diferente manera, dependiendo de sus hábitos de vida, las circunstancias que hayan vivido y el entorno en el que se desarrolla. No obstante, en todos los casos genera desventajas o debilidades en el que "deteriora una serie de funciones" en diferentes ámbitos como el hogar, la sociedad y sobre todo en la persona en sí misma, más si no se encuentra preparada para afrontar esta etapa de su vida. ${ }^{14}$

En la rama de la medicina como en la psicología está comprobado que existe una diferencia en la forma que se presenta el envejecimiento en cada persona; es decir, una persona puede contar con sus capacidades tanto de goce como de ejercicio hasta avanzada edad, y otras que por el paso del tiempo presentan más dificultad de por "afecciones severas como la demencia $u$ otras enfermedades"; es decir, en el ámbito medico se basan en un concepto biológico al definirlo como:

14 Coto Edwin; Jiménez, Yeimi y Núñez, Miriam, "La actividad física para el adulto mayor en el medio natural", InterSedes: Revista de las Sedes Regionales; vol. XIV, num.27, 2013, p. 170. Recuperado el 15 de septiembre de 2021 de https://www.redalyc.org/pdf/666/66627452009.pdf 
"El cambio gradual en un organismo que conduce a un mayor riesgo de debilidad, enfermedad y muerte. Tiene lugar en una célula u órgano o el organismo total en el lapso de vida de un adulto o cualquier ser vivo. Hay una disminución en las funciones biológicas y en la capacidad para adaptarse al estrés metabólicos. Cambios en órganos que incluyen el remplazo de las células funcionales cardiovasculares con tejido fibroso. Efectos generales del envejecimiento incluyen inmunidad reducida, pérdida de fuerza muscular, disminución de la memoria y otros aspectos de la cognición y la perdida del color en el cabello y la elasticidad en la piel".

Mientras que en el ámbito medico por parte del psicólogo alemán Paul Baltes, uno de los autores más representativos de la psicología del transcurso vital, consideró al envejecimiento "como el estudio de la constancia y cambio en el comportamiento durante el transcurso de la vida". ${ }^{16}$

Por lo que resulta indispensable que el ámbito de las ciencias sociales se realice grandes esfuerzos por fomentar la eliminación hacia los estigmas sociales que posicionan al AM de

\footnotetext{
15 Alvarado Alejandra y Salazar Angela, "Análisis del concepto envejecimiento". Revista Scielo, vol. 25, num.2, 2014. Recuperado el 22 de enero de 2022 de: https://scielo.isciii.es/ pdf/geroko/v25n2/revision1.pdf

16 Delcey-Ruiz, Elisa, "Psicología social del envejecimiento y perspectiva del transcurso de la vida: consideraciones críticas", Revista Colombiana de Psicología, vol. 19, no. 2, julio-diciembre 2010. Recuperado el 22 de enero de 2022 de: http://www.scielo.org.co/pdf/rcps/v19n2/v19n2a05.pdf
}

manera generalizada. ${ }^{17}$ Es decir, puede tener deficiencias, cambios y complicaciones propios del envejecimiento, lo cual puede ocasionar que se ubique en una absoluta exclusión social, abandono, una baja autoestima e incluso la depresión, dado que su participación en distintas actividades, así como en la toma de decisiones se ve disminuida.

Culturalmente, la edad cronológica constituye un parámetro determinante sobre el inicio de la vejez, en base a ello se determina la productividad y capacidad del AM en relación con su persona y desarrollo con la sociedad. Por tal razón el derecho de cada persona a mejorar sus condiciones de vida no debe estar sujeto a un límite de edad, no obstante, para el adulto mayor maduro representa la oportunidad de una participación social.

\section{INICIO FISIOLÓGICO DE LA ETAPA DE LA VEJEZ: PREJUICIOS SOCIALES DE LA CAPACIDAD JURÍDICA EN LA VEJEZ}

El ser humano desarrolla su máxima capacidad fisica en la década comprendida entre los 20 y los 30 años, es a partir de los 30 años donde se presentan cambios en la masa y fuerza muscular, sin embargo, a partir de los 50 años es más evidente los cambios en la fuerza y masa muscular, aproximadamente del $15 \%$ por década. Por lo tanto, entre los 40 y 60 años el envejecimiento genera su pérdi-

17 Castellón, Gisela; González, Belkis; González, Caridad y Martínez, Teresita, "El envejecimiento, la vejez y la calidad de vida: ¿éxito o dificultad?", Revista Scielo, Finlay, vol. 8, no. 1 enero-marzo 2018. Recuperado el 19 de septiembre de 2021 de: http://scielo.sld.cu/scielo.php?script=sci_arttext\&pi$d=S 2221-24342018000100007$ 
da. ${ }^{18}$ Este cambio fisiológico forma parte del proceso del envejecimiento, sin embargo, no es una causal de impedimento que influya en la toma de decisión del adulto mayor maduro, por lo que se puede interpretar que desde el nacimiento el proceso del envejecimiento empieza a percibirse a simple vista.

La flexibilidad, es una cualidad esencial para una buena condición fisica al requerir movilidad articular y flexibilidad muscular, lo que permite una mayor autonomía. Por lo tanto, es esencial para cualquier individuo incluyendo a la persona materia de estudio en su día a día, por lo que la reducción de ésta complica la realización de movimientos como agacharse para ponerse un zapato, vestirse, poner y quitar un objeto en un lugar alto. ${ }^{19}$ De todo ello, cabe señalarse que para la persona que envejece, los cambios que se presentan no es algo que pueda ocultarse demasiado tiempo. En el AM, se presenta una importante disminución del movimiento articular, la cual depende de la información propioceptiva que provienen de los músculos, de los ligamentos y de las terminaciones nerviosas tanto de ella

18 Calero-Saa Pedro y Chaves, Marco, "Cambios fisiológicos de la aptitud física en el envejecimiento", Revista de Investigación en Salud. Universidad de Boyocá, vol.3, no.2, julio-diciembre 2016, p.182. Recuperado el 01 de octubre de 2021 de: https://revistasdigitales.uniboyaca.edu.co/index. $\mathrm{php/rs/article/view/178/195}$

19 Borba, Claudio; Coelho, Moisés; Gomes, Rodrigo y Oliveira, Marco, "Efectos de un programa de entrenamiento concurrente sobre la fuerza muscular, flexibilidad y autonomía funcional de mujeres mayores", Revista Redalyc Ciencias de la Actividad Fisica, vol. 15, no.2, julio-diciembre 2014. Recuperado el 01 de octubre de 20221 de: https://www.redalyc. org/articulo.oa?id=525652729002 misma como del ligamento periodontal y de la mucosa bucal, por lo tanto, en el caso del AM, su actividad propioceptiva está disminuida y lo que produce una disminución del control de los movimientos de la articulación. ${ }^{20}$ Por estas razones el AM, presenta mayor dificultad al ejercer su capacidad de goce, pues entre ellos se encuentra la disminución de movilidad al realizar ejercicio, caminar, correr o subir escaleras.

Los gerontólogos estiman que solamente un $30 \%$ del deterioro físico de la vejez tiene una base genética; el otro $70 \%$ está relacionado con factores conductuales y psicológicos, condiciones que pueden prevenirse o tratarse, por ejemplo ${ }^{21}$ : La apariencia de senilidad de algunas personas es el resultado de la prescripción de medicamentos inadecuados, combinaciones contraproducentes de ellos o incluso excesos en la ingestión de pastillas; la depresión y la pasividad son, frecuentemente, el resultado de la pérdida de la actividad relevante, de la estimulación intelectual y del control sobre los acontecimientos; y la debilidad y la fragilidad están causadas por un estilo de vida sedentario. El ejercicio y el entrenamiento ligero con pesas pueden restaurar la fuerza y la flexibilidad.

20 Cabrera, Marlene y Torre, Yaiset, "Desajustes intraarticulares en el paciente geriátrico", Revista Scielo, vol.20 no.2., mayo-agosto 2018. Recuperado el 01 de octubre de 2021 de: http://scielo.sld.cu/scielo.php?script=sci_arttext\&pi$d=S 1608-89212018000200069$

21 Mátas, Mercedes, "Desarrollo de los prejuicios sociales", Recuperado el 9 de octubre de 2021 de: https://www.um.es/ sabio/docs-cmsweb/aulademayores/tema_7_prejuicios desarrollo_0.pdf 
Como es indicado, la capacidad funcional se basa en las actividades de la vida diaria (AVD) como caminar o comer y las actividades instrumentales de la vida diaria (AIVD) como ir de compras o preparar una comida, por ende, conocer cuáles son las actividades y las limitantes que tiene en cada una de ellas o dificultad que puede presentar, se advierte que se establezcan variables que determinen el grado de funcionalidad del adulto, como se establecen con lo siguiente: Completamente capaces aquellas personas que se manejan en forma totalmente independiente; es decir, no tienen dificultad para realizar las actividades cotidianas, tanto elementales como instrumentales; Los levemente incapaces, que son aquellas personas que tienen dificultad sólo en las AIVD, sin importar su cantidad; Los moderadamente incapaces a aquellas personas que tienen dificultad en alguna de las AVD y además tienen dificultad en ninguna o una AIVD; Los severamente incapaces las personas que declararon tener dificultad en una o más AVD y en más de una de las AIVD, excluyendo a los que tienen dificultad en todas las AVD y las AIVD; y por último completamente incapaces a aquellas personas que tienen dificultad en todas las AVD y en todas las AIVD. ${ }^{22}$

Derivado de lo anterior, podemos destacar que, así como se clasifica la vejez en diferentes etapas por edades, de igual forma es por capacidades, por lo cual no es conveniente

22 González, César; Ham-Chanden, Roberto (2007), "Funcionalidad y salud; una tipología del envejecimiento en México", Revista Scielo Salud Pública de México, v.14 supl.4. Recuperado el 29 de septiembre de 2021 de: http://www.scielo.org.mx/scielo.php?script=sci_arttext\&pi$d=S 0036-36342007001000003$ generalizar a los que tienen más de 60 años, por ende, podemos destacar que la clasificación es de gran relevancia, pues aun cuando existe tal modelo, existe una necesidad de instrumentar diversas reformas en las políticas públicas para otorgar una atención adecuada a cada uno de los subgrupos que se han planteado.

Ahora bien, existe una tendencia implícita y explícita de algunos profesionales de la salud a suponer que no vale la pena gastar recursos en la atención de las personas adultas mayores, debido a que no cumplirán con los tratamientos prescitos, que tienen una patología crónica muy avanzada y que solo generan problemas a la familia y al personal de salud que los atienden, teniendo como consecuencia conductas ofensivas, actitudes que bloquean su atención y abandono en el manejo asistencial y hospitalario, negándoles un trato digno y la búsqueda de la calidad de vida en padecimientos crónicos. ${ }^{23}$

Lo anterior demuestra que la discriminación que se da hacia estos sujetos va en contra de la justicia al no permitir que puedan satisfacer sus necesidades básicas en igualdad de oportunidades que las otras etapas como la adultez, lo cual origina consecuencias que afectan tanto en al sujeto en lo individual como en su desarrollo con la sociedad. Es el resultado de una serie

23 Fajardo-Ortiz, Guillermo y Olivares-Santos, Roberto, "Viejismo en el ambiente cotidiano". Recuperado el 9 de octubre de 2021 de: http://inger.gob.mx/pluginfile.php/96260/mod_resource/content/355/Archivos/C_Promocion/Unidad_1/Lecturas_Complementarias/Viejismo\%20en\%20el\%20ambiente\%20cotidiano.pdf 
de prejuicios sociales y legales que se tiene acerca de una persona adulta mayor o de un determinado grupo, en este caso por pertenecer a la etapa de la vejez, porque no son las características propias con las que cuenta el individuo, sino a su identificación al asociarlo a esa etapa o identificarlo como integrante de un grupo determinado como la vulnerabilidad.

Por su parte, la OMS ${ }^{24}$ ha indicado que el maltrato en las instituciones puede consistir en inmovilizar a los pacientes, tratarlos de forma vejatoria (por ejemplo, dejándoles con ropa sucia) o no dejarles tomar decisiones en relación con asuntos cotidianos. atenderlos insuficientemente de forma intencional (como dejando que desarrollen úlceras de decúbito); medicarlos de forma excesiva o no suficiente, o impedirles que tomen medicamentos, y tratarlos de forma negligente y abusiva desde un punto de vista emocional.

El prejuicio no es solamente una declaración de opinión o de creencia, sino una actitud que incluye sentimientos tales como desprecio, disgusto o total repudio. ${ }^{25}$ Esa actitud se basa en ideas preconcebidas en cada mente humana, la cual la mayoría de las veces son falsas, pues le resulta más fácil dar por sentado un

24 Organización Mundial de la Salud, Centro de prensa, "Maltrato de las personas mayores" del 15 de junio de 2021. Recuperado el 9 de octubre de 2021 de: https://www.who.int/ es/news-room/fact-sheets/detail/elder-abuse

25 Casas Martínez, María de la Luz, "Prejuicios, estereotipos y discriminación. Reflexión ética y psicodinámica sobre la selección de sexo embrionario", Revista Acta Bioethica, vol. 14 no. 2, 2008, p. 151. Recuperado el 29 de septiembre de 2021 de: https://www.redalyc.org/pdf/554/55412249004.pdf sinnúmero de situaciones que reflexionar sobre ello.

Los avances económicos y sociales han permitido en el mundo contar con servicios médicos modernos, antibióticos, programas masivos de inmunización, agua potable y drenaje, mismos que ayudan a reducir la mortalidad infantil, a combatir infecciones y con ello a sobrevivir cada vez más para alcanzar las edades avanzadas. ${ }^{26}$ Derivado de lo anterior se puede deducir que el mundo está cambiando, y no es algo que se vaya a detener, el cambio es constante, y así como no imaginábamos los alcances de la medicina queda mucho por descubrir; por lo tanto, llegar a más de 60 años con una buena salud es posible, pues estos avances permiten que las personas puedan desarrollarse mejor de lo que uno cree.

\section{PREJUICIOS LEGALES: POLÍTICAS PÚBLICAS EN EL ÁMBITO MÉDICO}

Cuidar es una actividad propia y esencial de la humanidad para la conservación de la vida, no depende solo del ámbito médico y de su personal de la salud, el autocuidado es tan importante por lo cual resulta fundamental determinar cuáles son los elementos que en el ámbito médico se debe considerar para que la persona adulta mayor, sea considerado como un individuo con la aptitud de ejercer su derecho al desarrollo de la autonomía en la elección de cualquier decisión médica. Pese a

26 González, César y Ham, Roberto, “Discriminación en las edades avanzadas en México", Revista Scielo, vol.14, no.55, enero-marzo 2008. Recuperado el 29 de septiembre de 2021 de: http://www.scielo.org.mx/scielo.php?script=sci_arttext\&pid=S1405-74252008000100003 
que esa es una de las cuestiones más debatibles al posicionar al individuo en la situación donde no es tenido en cuenta en asuntos que tienen que ver con los cuidados médicos de su calidad de vida, por ende, tal importante es el respeto al principio de autonomía y autorrealización, asimismo como al principio de la dignidad humana.

En esta línea, Bach y Kerzner señalan que la definición de capacidad jurídica desde el modelo social no se concentra en los atributos o limitaciones individuales de la persona, sino más bien en las barreras sociales, económicas y legales que la persona enfrenta a la hora de formular o ejecutar decisiones personales, así como, en el apoyo y los ajustes que requieren ser dados para facilitar la toma de decisiones. ${ }^{27}$ Por lo tanto, los adultos mayores requieren una serie de cuidados derivados de la edad de su organismo que con el tiempo se debilita, e incluso lleva a que la persona dependa de otras para realizar actividades de la vida cotidiana.

Lo anterior es un fenómeno reconocido legalmente, y por ello desde distintas autoridades en salud se ha venido trabajando por mejorar la atención que les brinda el sistema de salud, lo cual compromete a sus familias y cuidadores, sin embargo, no siempre es posible que estos últimos asuman su responsabilidad y por

27 Villarreal, Carla, "El reconocimiento de la capacidad jurídica de las personas con discapacidad mental e intelectual y su incompatibilidad con los efectos jurídicos de la interdicción y la curatela: Lineamientos para la reforma del Código Civil y para la implementación de un sistema de apoyos en el Perú", marzo 2014. Recuperado el 9 de octubre de 2021 de: https:// www.corteidh.or.cr/tablas/r36872.pdf. ende se aprecian casos de abandono, un problema que pone en riesgo la vida, salud, integridad y bienestar del adulto mayor. También puede presentarse el maltrato y negligencia en personas mayores el cual ha sido definido por la Asociación Médica como aquel acto u omisión que provoca en el anciano daño o temor hacia su salud o bienestar que se realiza en el momento o por medio de amenazas. ${ }^{28}$

Al respecto el Código de Ética Médica Internacional establece que "el médico debe dedicarse a proporcionar un servicio médico competente, con plena independencia profesional y moral, con compasión y respeto por la dignidad humana". ${ }^{29}$ A pesar de esto, en la actualidad es evidente la falta de regulación por fortificar tal relación, al permitir la exclusión del adulto mayor en las decisiones médicas. La Ley Estatal de Salud en Nuevo León, no nos brinda elementos que en el ámbito médico debe regir a todo personal de la salud con referencia a las personas adultas mayores, al respecto solo menciona en el artículo 4 II Bis que "corresponde al Estado en materia de salubridad general, la atención médica a las personas adultas mayores en áreas de salud gerontológica y geriátrica". ${ }^{30}$ Al contra-

28 Giraldo Rodríguez, Liliana (2015); "El maltrato de las personas de edad", Ciencias Médicas del Instituto Nacional de Geriatría, Pág. 231-232, Recuperado el 18 de septiembre de 2021 de: http://www.geriatria.salud.gob.mx/descargas/publicaciones/Envejecimiento_y_salud_3a_edicion.pdf

29 H. Congreso del Estado de Nuevo León (2021), "Ley Estatal de Salud", Recuperado el 26 de septiembre de 2021 de: http://www.hcnl.gob.mx/trabajo_legislativo/leyes/leyes/ ley_estatal_de_salud/

30 H. Congreso del Estado de Nuevo León, "Ley Estatal de Salud" Recuperado el 09 de octubre de 2021 de: http://www. 
rio de la Carta de los Derechos Generales de los Pacientes, donde el decálogo incluye en cada uno de sus artículos de los derechos a de cada uno de los pacientes, así como el fundamento legal de la Ley General de Salud que se relaciona con el ámbito médico.

De acuerdo con la Página Oficial del Gobierno del Estado de Nuevo León los derechos de los pacientes son los siguientes:

1. Recibir atención médica adecuada;

2. Recibir trato digno y respetuoso;

3. Recibir información suficiente, clara, oportuna y veraz;

4. Decidir libremente sobre su atención;

5. Otorgar o no su consentimiento válidamente informado;

6. Ser tratado con confidencialidad;

7. Contar con facilidades para obtener una segunda opinión;

8. Recibir atención médica en caso de urgencia;

9. Contar con un expediente clínico; y,

10. Ser atendido cuando se inconforme por la atención médica recibida. ${ }^{31}$

El consenso de la Asociación Internacional de Psicogeriatría (IPA) definió la población en grupos de edades para la aplicación de diagnósticos, tratamientos, programas de desarroIlo, con el fin de ofrecer un mejor manejo de la siguiente manera: adulto mayor joven 55-64 años, adulto mayor maduro 65-74 años, adulto mayor 75-84 años, ancianos mayores de

hcnl.gob.mx/trabajo_legislativo/leyes/leyes/ley_estatal_de_ salud/

31 Gobierno del Estado de Nuevo León (2015), "Conoce tus derechos". Recuperado el 30 de septiembre de 2021 de https://www.saludnl.gob.mx/drupal/conoce-tus-derechos.
85 años, nonagenarios y centenarios. ${ }^{32}$ Ante esta clasificación, resulta una realidad la necesidad de definir que, a pesar de ellas, debe existir una gran colaboración para comprender cada situación de AM, promover sus derechos sociales y contribuir a su desarrollo en acciones para la mejora tanto personal como social.

Otorgar una información adecuada a las personas adultas mayores se cumple al considerar que cada uno es diferente, debido a que no todos se encuentran en la misma situación biológica y física. Conforme al artículo 4, fracción $X$ nos define a la elaboración de un Estándar "al documento técnico que prevé un uso común y repetido de reglas, especificaciones, atributos o métodos de prueba aplicables a un bien, producto, proceso o servicio, así como aquéllas relativas a terminología, simbología, embalaje, marcado, etiquetado o concordaciones". Por lo que una acción de responsabilidad médica es asegurar que el AM entienda su estado de salud, las alternativas para recibir un tratamiento médico, las consecuencias de dicho tratamiento, es decir, toda información que se le brinde debe ser lo más clara posible para que el adulto comprenda su situación, de ese modo podrá ejercer su autonomía al tomar la decisión por su cuenta, pensar en sus alternativas y poder expresarla libremente. ${ }^{3}$

2 García, Lena y García, Luis; "El adulto mayor maduro: condiciones actuales de vida", Revista Médica de Risaralda Dialnet, vol. 11, no. 2, 2005. Recuperado el 29 de septiembre de 2021 de: Dialnet-EIAdultoMayorMaduro-5030408 (2).pdf 33 Barrante, Melba; Lama, Alexis y Rodríguez, Eduardo, "Relación Médico-Paciente: Derechos del adulto mayor" Revista Scielo Acta Bioética, vol.15, no.2, noviembre 2009. Recupera- 
Lo antes mencionado implica que la vejez es la etapa en la cual la persona AM sufre cambios verdaderamente significativos derivados del proceso de envejecimiento, al hacerse más notorio ante las personas, como la disminución de actividades físicas, su vista no es la misma o inclusive escuchar, todos estos cambios fisiológicos son parte de la vida. Por lo tanto, el servicio de salud y una seguridad social podría manifestarse como una de las principales necesidades que se presenta en el individuo al iniciar la etapa de la vejez.

A su vez, la Asociación Médica Mundial expone sus recomendaciones para que los médicos puedan dar un trato a los ancianos, asimismo exhorta a las asociaciones médicas nacionales a dar publicidad a la Declaración entre los miembros y ante la opinión pública. Conforme al Manual de Políticas de la AMM de dicho organismo en la Declaración de Hong Kong de la AMM sobre el maltrato de anciano, los médicos que atiendan al individuo antes mencionado deben seguir estas recomendaciones $^{34}$ :

- hacer mayores esfuerzos para crear una relación de confianza con los pacientes ancianos a fin de instarlos a pedir atención médica cuando sea necesario y a sentirse cómodos cuando confíen en el médico;

- proporcionar una evaluación y tratamien-

do el 18 de septiembre de 2021 de: https://www.scielo.cl/scielo. php?script=sci_arttext\&pid=S1726569X2009000200013\&In$\mathrm{g}=\mathrm{en} \& \mathrm{nrm}=\mathrm{iso} \& \operatorname{lng}=\mathrm{en}$

34 Asociación Médica Mundial, "Manual de Políticas de la AMM", diciembre 2011, p.59. Recuperado el 30 de septiembre de 2021 de: https://www.wma.net/wp-content/ uploads/2019/10/HB-S-Version-2019.pdf to médico por los daños producidos por el abuso y/o abandono;

- intentar establecer o mantener una relación terapéutica con la familia (por lo general, el médico es el único profesional que mantiene un contacto duradero con el paciente y la familia), y mantener en la máxima medida posible la confidencialidad del paciente;

- informar toda sospecha de casos de maltrato o abuso de ancianos, conforme a la legislación local; utilizar un equipo multidisciplinario de tratantes de las profesiones médica, servicio social, salud mental y legal, cada vez que sea posible;

- estimular la generación y utilización de recursos comunitarios de apoyo que presten servicios domiciliarios, reposo y disminución del estrés, a las familias de alto riesgo.

Por otro lado, el Código de Deontología Médica ha sido elaborado con la finalidad de ser el "conjunto de principios y reglas éticas que han de inspirar y guiar la conducta profesional del médico" (art. 1). ${ }^{35}$ Por lo tanto, existe un ordenamiento legal mediante el cual el médico que preste sus servicios para desarrollarse profesionalmente en el ámbito de la salud debe sujetarse a respetar y dar cumplimiento a su ética profesional; conforme a su artículo 12 , debe respetar los siguientes principios al ejercicio de su profesión:

1. El médico respetará el derecho del paciente a decidir libremente, después de recibir

35 Consejo General de Colegios Oficiales de Médicos, "Código de Deontología Médica, julio 2011. Recuperado el 9 de octubre de 2021 de: https://www.cgcom.es/sites/default/files/ codigo_deontologia_medica.pdf 
la información adecuada, sobre las opciones clínicas disponibles. Es un deber del médico respetar el derecho del paciente a estar informado en todas y cada una de las fases del proceso asistencial. Como regla general, la información será la suficiente y necesaria para que el paciente pueda tomar decisiones;

2. El médico respetará el rechazo del paciente, total o parcial, a una prueba diagnóstica o a un tratamiento. Deberá informarle de manera comprensible y precisa de las consecuencias que puedan derivarse de persistir en su negativa, dejando constancia de ello en la historia clínica;

3. Si el paciente exigiera del médico un procedimiento que éste, por razones científicas o éticas, juzgase inadecuado o inaceptable, el médico, tras informarle debidamente, quedará dispensado de actuar;

4. Cuando el médico atienda a una persona en huelga de hambre, le informará sobre las consecuencias del rechazo a la alimentación, así como de su previsible evolución y pronóstico. Respetará la libertad de quienes decidan de forma consciente y libre realizar huelga de hambre, incluidas las personas privadas de libertad, pudiendo acogerse a la objeción de conciencia si fuese obligado a contrariar esta libertad. ${ }^{36}$

Pero ocurre que los prejuicios legales ubican a una persona que solo por tener 60 años es perteneciente como grupo vulnerable conforme a la Ley de los Derechos de las Personas

36 Consejo General de Colegios Oficiales de Médicos, "Código de Deontología Médica, julio 2011. Recuperado el 9 de octubre de 2021 de: https://www.cgcom.es/sites/default/files/ codigo_deontologia_medica.pdf
Adultas Mayores del Estado de Nuevo León, y al referirnos a esa categoría se considera que no es apto para ejercer sus derechos y obligaciones legales. Por tanto, "Cuando el médico trate a pacientes incapacitados legalmente $o$ que no estén en condiciones de comprender la información, decidir o dar un consentimiento válido, deberá informar a su representante legal o a las personas vinculadas por razones familiares o de hecho" ${ }^{37}$, pero también nos señala que "El médico deberá ser especialmente cuidadoso para que estos pacientes participen en el proceso asistencial en la medida que su capacidad se lo permita". ${ }^{38}$

No obstante, lo expuesto por el artículo 13 como se ha discutido, no todos los adultos mayores se encuentran con una disminución de sus facultades para poder ejercer su autonomía, por lo tanto, a ese tipo de pacientes AMM, que se encuentre con plena capacidad de ejercicio, se le deben de hacer adaptables las reglas que se establecen en el artículo 12 del Código de Deontología Médica. Lo cual demuestra la falta necesaria de hacer ajustables tales reglas en el Estado de Nuevo León, instruir a cada médico los elementos necesarios para valorar a los adultos mayores, y hacer la clara distinción que un AMM donde se estima entre los años 65 a 74 años, sí han disminuido sus facultades, pero no lo ubican

37 Consejo General de Colegios Oficiales de Médicos, "Código de Deontología Médica, julio 2011. Recuperado el 9 de octubre de 2021 de: https://www.cgcom.es/sites/default/files/ codigo_deontologia_medica.pdf

38 Consejo General de Colegios Oficiales de Médicos, "Código de Deontología Médica", julio 2011. Recuperado el 9 de octubre de 2021 de: https://www.cgcom.es/sites/default/files/ codigo_deontologia_medica.pdf 
como un todo incapaz de ejercer sus derechos y obligaciones legales. De este modo se daría cumplimiento a lo que se establece en el artículo $1^{\circ}$ fracción III de la Ley de Infraestructura de la calidad "Propiciar la innovación tecnológica en los bienes, productos, procesos y servicios para mejorar la calidad de vida de las personas en todo el territorio nacional".

No todos los adultos mayores presentan padecimientos, hay quienes cuentan con una salud que no presenta alguna incapacidad, lo único que está presente es el deterioro proveniente de la edad. Por lo cual, es responsabilidad del Estado conforme al artículo 48 de la Ley de Personas adultas Mayores del Estado de Nuevo León cumplir con lo siguiente:

"Las instituciones públicas y privadas, casas hogar, albergues, residencias de día o cualquier otro centro de atención a las personas adultas mayores, deberán ajustar su funcionamiento a lo dispuesto por las Normas Oficiales Mexicanas, Normas Mexicanas, Normas Técnicas y los reglamentos que se expidan para este efecto". ${ }^{40}$

Por este motivo, la evaluación geriátrica inte-

39 Cámara de Diputados del H. Congreso de la Unión, "Ley de Infraestructura de la Calidad" del 1 de julio de 2021. Recuperado el 10 de octubre de 2021 de: http://www.diputados. gob.mx/LeyesBiblio/pdf/LICal_010720.pdf

40 Congreso del Estado de Nuevo León, “Ley de los Derechos de las Personas Adultas Mayores en el Estado de Nuevo León", Recuperado el 27 de septiembre de 2021 de: http:// www.hcnl.gob.mx/trabajo_legislativo/leyes/leyes/ley_de_los_ derechos_de_las_personas_adultas_mayores_en_el_estado_de_nuevo_leon gral, la medición de la capacidad funcional, la calidad de vida y el autocuidado resulta de tan importante trascendencia.

\section{PRINCIPIO DE DIGNIDAD HUMANA, AUTONOMÍA Y AUTORREALIZACIÓN DEL ADULTO MAYOR MADURO EN LA VEJEZ:}

Tomando de referencia al autor Pablo Simón-Lorda ${ }^{41}$, el ejercicio de la autonomía de las personas, de los pacientes, exige que se cumplan al menos tres condiciones:

1. Actuar voluntariamente, es decir libre de coacciones externas

2. Tener información suficiente sobre la decisión que va a tomar, es decir, sobre el objetivo de la decisión, sus riesgos, beneficios y alternativas posibles.

3. Tener capacidad, esto es, poseer una serie de aptitudes psicológicas (cognitivas, volitivas y afectivas) que le permiten conocer, valorar y gestionar adecuadamente la información anterior, tomar una decisión y expresarla.

Por tanto, se considera que un paciente ejerce el derecho a su autonomía cuando por su cuenta toma las decisiones pertinentes a su persona, al expresar su voluntad basándose en las recomendaciones médicas al considerar sus opciones, y las consecuencias de cada elección que elija.

Simón-Lorda, Pablo, "La capacidad de los pacientes para tomar decisiones: una tarea todavía pendiente", Revista de la Asociación Española de Neuropsiquiatría", vol. 28, no.2, 2008. Recuperado el 29 de septiembre de 2021 de: https://scielo.isciii.es/scielo.php?script=sci_arttext\&pi$d=S 0211-57352008000200006$ 
De acuerdo con los autores Herrera y Guzmán, la ética médica le interesa la idea de dignidad de la vida, especialmente en lo que atañe a la relación médico-paciente; es indispensable tenerla presenta al decidir entre la vida y la muerte en situaciones en que la vida no puede prolongarse indefinidamente y en cualquier condición; esto es, cuando ha perdido su dignidad intrínseca. ${ }^{42}$ Conforme lo expuesto por los autores aludidos, demuestra que una persona al entrar a la etapa de la vejez no significa que haya adquirido una mentalidad envejecida, pues bien puede contar con capacidad legal, aun cuando por cambios fisiológicos necesite más atención, cuidados o servicios a los que tiene derecho, sin embargo, no siempre los recibe. A su vez demuestra que se están violentando los derechos de los adultos mayores al no garantizarles un desarrollo a su vida digna.

De manera que, valorar sistemáticamente la capacidad sensorial y funcional del adulto como paciente, puesto que al tener en cuenta sus limitaciones se puede mejorar o adaptar las medidas necesarias. Asimismo, con la capacidad funcional, dado que al incluir en la historia clínica la valoración del estado físico, es decir, la capacidad de realizar las actividades básicas e instrumentales de la vida diaria

42 Herrera, Adela y Guzmán, Andrés, "Reflexiones sobre la calidad de vida, dignidad y envejecimiento", Revista Médica Clínica Condes, vol. 23, no.1, enero 2012, p.67. Recuperado el 29 de septiembre de 2021 de: https://reader. elsevier.com/reader/sd/pii/S0716864012702754?token=2FD46C497019927EEA1331F90605499C898612C581970 5877B1A89DD3EAEAB9C499D98599554DC554A0085CC7E3CA9D1\&originRegion=us-east-1\&originCreation $=20211007020734$ y su estado mental que es su capacidad cognitiva y afectiva se consigue conocer más en profundidad al paciente y poder ajustar las recomendaciones a su situación real y mejorar así su cumplimiento. ${ }^{43}$

Logrando este propósito, no existe duda alguna que se respeta el derecho social, dado que tanto el personal médico como el Estado con la creación de las debidas políticas públicas, otorgan una mayor y efectiva atención para el grupo de adultos mayores, procurando en todo momento que el valor moral se cumpla y al fortalecimiento de una actitud de respeto por cualquier institución.

Por lo tanto, hace falta una mayor inclusión del AM, que como se ha demostrado, no todos envejecen de la misma forma. Un ajuste de adaptación a las políticas públicas, en el cual los que pertenecen al ámbito de la salud puedan considerar para determinar si el individuo es capaz de ejercer su autonomía en la elección de cuestiones médicas, ponen en manifiesto que la edad no debe ser una excusa para para justificar tal exclusión.

La competencia que es el término legal de capacidad de decisión utiliza diversos criterios: habilidad para comunicar la elección, comprensión de la información sobre tratamiento, apreciación de la situación y sus consecuencias. ${ }^{44}$ Por lo tanto, las personas que se en-

43 Serra Rexach, JA “Comunicación entre el paciente anciano y el médico", Revista Scielo Anales de Medicina Interna, vol.20 no.2, febrero 2003. Recuperado el 29 de septiembre de 2021 de: https://scielo.isciii.es/scielo.php?script=sci_arttext\&pid=S0212-71992003000200001

44 Cárdenas, Maximiliano; Delgado Ana; Sogi, Cecilia y Za- 
cuentran en la tercera edad pueden desarrollarse como personas independientes con la creación de medidas necesarias para que puedan lograr desenvolverse en cualquier entorno en que se encuentre.

Estimulando estas acciones se logra que el AM se sienta capaz de lograr una vida plena, al adaptarse a su situación, reconociendo sus limitaciones de acuerdo con sus condiciones, capacidades y medios, que con un trato digno es capaz de reconocer. Lo cual demuestra que estas personas tienen los mismos derechos y obligaciones que cualquier otro ser humano, por tal razón es importante que se le considere sujeto con capacidad legal para ejercerlo a través de medios dignos.

A pesar de lo estipulado en la Declaración Universal de Derechos Humanos ${ }^{45}$, al definir que todos los seres humanos son iguales en dignidad y derechos, es decir desde la etapa de la infancia ya la persona ha aprendido a diferenciarse y a los que lo rodean, siempre con sus criterios que suelen estar basados en sus características físicas como el sexo, la raza, nacionalidad, orientación sexual u otros, o basados en la condición de edad de la persona, es decir al caracterizarlo por la etapa

vala, Salomón, "Autonomía del paciente y toma de decisiones en salud: conocimiento en internos de medicina - 2010", Revista Scielo Anales de la Facultad de Medicina, vol.73 no.1, enero-marzo 2012. Recuperado el 29 de septiembre de 2021 de: http://www.scielo.org.pe/scielo.php?script=sci_arttext\&pi$d=S 1025-55832012000100004$

45 Organización de las Naciones Unidas, "Declaración Universal de los Derechos Humanos". Recuperado el 30 de septiembre de 2021 de: https://www.un.org/es/documents/udhr/ UDHR_booklet_SP_web.pdf biológica en la que se encuentra. De manera que al considerar diferentes a los demás se está respetando las diferencias que existen entre cada ser humano, sin dejar a un lado que aquellos a quienes ha considerado diferentes sean mejores unos de otros, o que tengan unos más o con menos derechos.

La dignidad humana se presenta desde una concepción católica tradicional, la dignidad común a todos los seres humanos procede de su condición de hijos de Dios y reside en la capacidad de acatar y observar la ley moral, la cual de ninguna manera emana de los humanos mismos, sin embargo, para otros, por el contrario, la dignidad humana consiste en la capacidad que tenemos los humanos de darnos ley moral a nosotros mismos. ${ }^{46}$ Esta es la finalidad de la cual debe enfocarse el criterio en el ámbito médico, en lograr el mejoramiento mediante reformas y en la práctica de la medicina para brindar una mejor atención a quienes están en la etapa de la vejez pues al presentar diversas dificultades por sobrevivir, se encuentra en gran desventaja.

El consentimiento informado, es un documento formal de relación entre los profesionales de salud y los pacientes, incluyendo sus familiares, donde su finalidad es el respeto por la autonomía ya autodeterminación del sujeto afecto por la enfermedad, por lo tanto, se puede decir que es el principio más importante en el proceso de la toma de decisión del paciente

46 Valls, Ramón, "El concepto de dignidad humana", Revista de Bioética y Derecho, no.23, diciembre 2011, p.1, Recuperado el 30 de septiembre de 2021 de: https://www.redalyc.org/ pdf/783/78339725004.pdf 
con el acompañamiento del médico. ${ }^{47}$ Por tanto, la salud del adulto depende en gran medida de que este sea tratado y respetado con dignidad, independientemente de las capacidades que sufre, que por lo general son propias de la edad, por lo que al no existir prueba que demuestre lo contrario, toda información otorgada debe manifestarse con tal claridad dependiendo sus cualidades para poder tomar su propia elección, de tal modo respetando que pueda ejercer su autonomía.

\section{CONCLUSIÓN}

Nacer, crecer, morir es el proceso de la vida, por lo tanto, los cambios fisiológicos y biológicos como se expusieron en el trabajo, muestran que cada cambio en el ser humano se presenta de forma gradual, mediante el cual dichos cambios juegan un papel fundamental, y no por pertenecer a la etapa de la vejez solo por tener 60 años, lo convierte en un ser sin aptitud de ejercer los derechos como paciente aludidos líneas atrás, conforme al principio de autonomía y autorrealización. Pues si bien, el adulto mayor maduro (AMM) no tiene la misma condición física que solía tener en su juventud, o capacidad de analizar de forma rápida lo que se le informa, un AMM aún conserva ciertas facultades que lo hacen apto con las medidas adecuadas a comprender el mundo que lo rodea.

Los prejuicios legales que existen en las políticas públicas son notorios, ninguna ha puesto

47 Burdiles, Patricio y Ortiz, Armando, "Consentimiento Informado", Revista Médica Clínica Condes, vol. 21, no. 4, julio 2010, p. 644. Recuperado el 30 de septiembre de 2021 de: https://www.elsevier.es/es-revista-revista-medica-clinica-las-condes-202-pdf-S0716864010705824 un énfasis en la protección que requiere cada una de las clasificaciones que dentro de la vejez existen. Pues si bien, lo que tienen en común es que al pertenecer al grupo vulnerable necesitan de una mayor protección jurídica, ninguna ha logrado determinar que, frente a sus disminuciones corporales, son sujetos con capacidad de entender con la adecuación de los medios necesarios en ellos.

Por su parte, es importante respetar el derecho a la dignidad humana que evidentemente se viola al hacer uso de los prejuicios sociales para no respetar al adulto mayor maduro en las cuestiones sobre su salud. Se demuestra que tales prejuicios influyen en el ámbito médico, al no respetar los "derechos de los pacientes" que se declaran en la Secretaría de Salud del Estado de Nuevo León, pues al catalogar a una persona como "viejo" no le brindan el respeto al no considerarlo apto para ejercer sus derechos conforme al principio de autonomía. Por lo anterior, se propone capacitar a las instituciones públicas, para que mediante protocolos, reglamentación interna y adiestramientos puedan brindar una mejor calidad de vida en el individuo sujeto de esta investigación, mejorando de este modo el servicio de salud a brindar. Que acorde al bioderecho, se respeten los principios de cada ser humano, como los de la materia de este estudio el principio de autonomía y autorrealización igualmente el de la dignidad humana de manera legal, desde un punto de vista ético.

\section{REFERENCIAS:}

Alvarado Alejandra y Salazar Angela (2014), "Análisis del concepto envejecimiento". Revista Scielo, vol. 25, no. 2, 2014. Recuperado el 22 de enero de 2022 de: https://scie- 
lo.isciii.es/pdf/geroko/v25n2/revision1.pdf

Asociación Médica Mundial, "Manual de Políticas de la AMM", diciembre 2011, p.59. Recuperado el 30 de septiembre de 2021 de: https://www.wma.net/wp-content/ uploads/2019/10/HB-S-Version-2019.pdf

Barrante, Melba; Lama, Alexis y Rodríguez, Eduardo, "Relación Médico-Paciente: Derechos del adulto mayor" Revista Scielo Acta Bioética, vol.15, no.2, noviembre 2009. Recuperado el 18 de septiembre de 2021 de: https://

Borba, Claudio; Coelho, Moisés; Gomes, Rodrigo y Oliveira, Marco, "Efectos de un programa de entrenamiento concurrente sobre la fuerza muscular, flexibilidad y autonomía funcional de mujeres mayores", Revista Redalyc Ciencias de la Actividad Fisica, vol. 15, no.2, julio-diciembre 2014. Recuperado el 01 de octubre de 20221 de: https://www. redalyc.org/articulo.oa?id=525652729002

Burdiles, Patricio y Ortiz, Armando, "Consentimiento Informado", Revista Médica Clínica Condes, vol. 21, no. 4, julio 2010, p. 644. Recuperado el 30 de septiembre de 2021 de: https://www.elsevier.es/es-revista-revista-medica-clinica-las-condes-202-pdf-S0716864010705824

Cabrera, Marlene y Torre, Yaiset, "Desajustes intraarticulares en el paciente geriátrico", Revista Scielo, vol.20 no.2., mayo-agosto 2018. Recuperado el 01 de octubre de 2021 de: http://scielo.sld.cu/scielo.php?script=sci_arttext\&pi$d=S 1608-89212018000200069$

Calero-Saa Pedro y Chaves, Marco, "Cambios fisiológicos de la aptitud física en el envejecimiento", Revista de Investigación en Salud. Universidad de Boyocá, vol.3, no.2, julio-diciembre 2016, p.182. Recuperado el 01 de octubre de 2021 de: https://revistasdigitales.uniboyaca.edu.co/index.php/rs/article/view/178/195

Cámara de Diputados del H. Congreso de la Unión, "Ley de Infraestructura de la Calidad" del 1 de julio de 2021. Recuperado el 10 de octubre de 2021 de: http://www.diputados.gob.mx/LeyesBiblio/pdf/LICal_010720.pdf

Cárdenas, Maximiliano; Delgado Ana; Sogi, Cecilia y Zavala, Salomón, "Autonomía del paciente y toma de decisiones en salud: conocimiento en internos de medicina - 2010", Revista Scielo Anales de la Facultad de Medicina, vol.73 no.1, enero-marzo 2012. Recuperado el 29 de septiembre de 2021 de: http://www.scielo.org.pe/scielo.php?script=sci_arttext\&pid=S1025-55832012000100004

Casas Martínez, María de la Luz, "Prejuicios, estereotipos y discriminación. Reflexión ética y psicodinámica sobre la selección de sexo embrionario", Revista Acta Bioethica, vol. 14 no. 2, 2008, p. 151. Recuperado el 29 de septiembre de 2021 de: https://www.redalyc.org/ pdf/554/55412249004.pdf

Castellón, Gisela; González, Belkis; González, Caridad y Martínez, Teresita, "El envejecimiento, la vejez y la calidad de vida: ¿éxito o dificultad?”, Revista Scielo, Finlay, vol. 8, no.1 enero-marzo 2018. Recuperado el 19 de septiembre de 2021 de: http://scielo.sld.cu/scielo.php?script=sci_arttext\&pid=S2221-24342018000100007

Comisión Nacional de los Derechos Humanos, "Los Derechos Humanos de las Personas Mayores". Recuperado el 9 de octubre de 2021 de: https://www.cndh.org.mx/sites/ all/doc/cartillas/2015-2016/27-DH-Adultos-Mayores.pdf.

Congreso del Estado de Nuevo León, "Ley de los Derechos de las Personas Adultas Mayores en el Estado de Nuevo León", Recuperado el 27 de septiembre de 2021 de: http://www.hcnl.gob.mx/trabajo_legislativo/leyes/leyes/ ley_de_los_derechos_de_las_personas_adultas_mayores_en_el_estado_de_nuevo_leon/

Consejo General de Colegios Oficiales de Médicos, "Código de Deontología Médica, julio 2011. Recuperado el 9 de octubre de 2021 de: https://www.cgcom.es/sites/default/ files/codigo_deontologia_medica.pdf

Coto Edwin; Jiménez, Yeimi y Núñez, Miriam, "La actividad física para el adulto mayor en el medio natural", InterSedes: Revista de las Sedes Regionales; vol. XIV, num.27, 2013, p. 170. Recuperado el 15 de septiembre de 2021 de https://www.redalyc.org/pdf/666/66627452009.pdf

Delcey-Ruiz, Elisa, "Psicología social del envejecimiento y perspectiva del transcurso de la vida: consideraciones críticas", Revista Colombiana de Psicología, vol. 19, no. 2, julio-diciembre 2010. Recuperado el 22 de enero de 2022 de: http://www.scielo.org.co/pdf/rcps/v19n2/v19n2a05.pdf Fajardo-Ortiz, Guillermo y Olivares-Santos, Roberto, "Viejis- 
mo en el ambiente cotidiano". Recuperado el 9 de octubre de 2021 de: http://inger.gob.mx/pluginfile.php/96260/ mod_resource/content/355/Archivos/C_Promocion/Unidad_1/Lecturas_Complementarias/Viejismo\%20en\%20 el\%20ambiente\%20cotidiano.pdf

Flores, Liliam, "Grupos Vulnerables", Recuperado el 26 de septiembre de 2021 de: http://archivos.diputados.gob.mx/ Centros_Estudio/Cesop/Eje_tematico_old_14062011/9_ gvulnerables_archivos/G_vulnerables/d_gvulnerables. $\mathrm{htm}$

García, Lena y García, Luis; "El adulto mayor maduro: condiciones actuales de vida", Revista Médica de Risaralda Dialnet, vol. 11, no. 2, 2005. Recuperado el 29 de septiembre de 2021 de: Dialnet-EIAdultoMayorMaduro-5030408 (2).pdf

Giraldo Rodríguez, Liliana (2015); "El maltrato de las personas de edad", Ciencias Médicas del Instituto Nacional de Geriatría, Pág. 231-232, Recuperado el 18 de septiembre de 2021 de: http://www.geriatria.salud.gob.mx/descargas/ publicaciones/Envejecimiento_y_salud_3a_edicion.pdf

Gobierno del Estado de Nuevo León (2015), “conoce tus derechos". Recuperado el 30 de septiembre de 2021 de https://www.saludnl.gob.mx/drupal/conoce-tus-derechos.

González, César y Ham-Chanden, Roberto (2007), “Funcionalidad y salud; una tipología del envejecimiento en México", Revista Scielo Salud Pública de México, v.14 supl.4. Recuperado el 29 de septiembre de 2021 de: http://www.scielo.org.mx/scielo.php?script=sci_arttext\&pi$\underline{d=S 0036-36342007001000003}$

González Jerónimo y Fuente Raquel, “Desarrollo humano en la vejez: un envejecimiento optimo desde los cuatro componentes del ser humano". INFAD Revista de Psicología, vol. 7, núm. 2, 2014. Recuperado el 22 de enero 2022 de: https://www.redalyc.org/pdf/3498/349851791013.pdf.

H. Congreso del Estado de Nuevo León (2021), "Código Civil para el Estado de Nuevo León", Recuperado el 26 de septiembre de 2021 de: http://www.hcnl.gob.mx/trabajo_legislativo/leyes/codigos/codigo_civil_para_el_estado_de_nuevo_leon/

H. Congreso del Estado de Nuevo León, "Ley de los Dere- chos de las Personas Adultas Mayores en el Estado de Nuevo León", Recuperado el 27 de septiembre de 2021 de: http://www.hcnl.gob.mx/trabajo_legislativo/leyes/ leyes/ley_de_los_derechos_de_las_personas_adultas_ mayores_en_el_estado_de_nuevo_leon/

H. Congreso del Estado de Nuevo León (2021), "Ley Estatal de Salud", Recuperado el 26 de septiembre de 2021 de: http://www.hcnl.gob.mx/trabajo_legislativo/leyes/leyes/ ley_estatal_de_salud/

Herrera, Adela y Guzmán, Andrés, "Reflexiones sobre la calidad de vida, dignidad y envejecimiento", Revista Médica Clínica Condes, vol. 23, no.1, enero 2012, p.67. Recuperado el 29 de septiembre de 2021 de: https:// reader.elsevier.com/reader/sd/pii/S0716864012702754?token=2FD46C497019927EEA1331F90605499C89861 2C5819705877B1A89DD3EAEAB9C499D98599554DC554A0085CC7E3CA9D1\&originRegion=us-east-1\&originCreation=20211007020734

Hernández, Mónica, "El Concepto de Discapacidad: De la enfermedad al enfoque del Derecho". Revista CES Derecho, vol.6, núm.2. Recuperado el 22 de enero de 2022 de: http://www.scielo.org.co/pdf/cesd/v6n2/v6n2a04.pdf\#: :text=En $\% 20 \%$ C3\%A9pocas $\% 20$ hist $\%$ C $3 \%$ B3ricas $\% 20$ la $\% 20$ discapacidad $\% 20$ fue $\% 20$ objeto $\% 20$ de,sus $\% 20$ facultades $\% 20$ intelectualesse $\% 20$ denominaban $\% 20$ \%E2\%80\%9Cmente $\% 20$ captus. $\%$ E2\%80\%9D $\% 20$ \%28Mu\%C3\%B1oz\%2C\%202010\%29

Instituto Nacional de Estadística y Geografía, "Censo de población y vivienda 2020, Comunicado de prensa num.24/21 del 25 de enero de 2021". Recuperado el 9 de octubre de 2021 de: https://www.inegi.org.mx/contenidos/ saladeprensa/boletines/2021/EstSociodemo/ResultCenso2020_Nal.pdf

Instituto Nacional de Estadística y Geografía, "Censo de población y vivienda 2020, Presentación de Resultados de Nuevo León”. Recuperado el 9 de octubre de 2021 de: https://www.inegi.org.mx/contenidos/programas/ccpv/2020/doc/cpv2020_pres_ res_nl.pdf

Mátas, Mercedes, "Desarrollo de los prejuicios socia- 
les", Recuperado el 9 de octubre de 2021 de: https:// www.um.es/sabio/docs-cmsweb/aulademayores/ tema_7_prejuicios_desarrollo_0.pdf

Organización de las Naciones Unidas, "Declaración Universal de los Derechos Humanos". Recuperado el 30 de septiembre de 2021 de: https://www.un.org/ es/documents/udhr/UDHR_booklet_SP_web.pdf

Organización Mundial de la Salud, "Demencia". Recuperado el 9 de octubre de 2021 de: https://www.who. int/es/news-room/fact-sheets/detail/dementia

Organización Mundial de las Naciones Unidas, "Informe mundial sobre el envejecimiento y la salud", Recuperado el 29 de septiembre de 2021 de: https://apps.who.int/iris/bitstream/handle/10665/186466/9789240694873_spa.pdf?sequence $=1$

Organización Mundial de la Salud, Centro de prensa, "Maltrato de las personas mayores" del 15 de junio de 2021. Recuperado el 9 de octubre de 2021 de: https://www.who.int/es/news-room/fact-sheets/detail/elder-abuse

Santofimio, German "De la anomalía a la discapacidad, una larga historia de exclusión social: De la muerte, al destierro y el repudio, a la inclusión educativa, Revista Inclusión y Desarrollo, vol. 3, no. 1, 2016. Recuperado el 22 de enero de 2022 de: http://biblioteca.uniminuto.edu/ojs/index.php/IYD/issue/archive Serra Rexach, JA "Comunicación entre el paciente anciano y el médico", Revista Scielo Anales de Medicina Interna, vol.20 no.2, febrero 2003. Recuperado el 29 de septiembre de 2021 de: https:// scielo.isciii.es/scielo.php?script=sci_arttext\&pi$d=S 0212-71992003000200001$

Simón-Lorda, Pablo, "La capacidad de los pacientes para tomar decisiones: una tarea todavía pendiente", Revista de la Asociación Española de Neuropsiquiatría", vol. 28, no.2, 2008. Recuperado el 29 de septiembre de 2021 de: https://scielo.isciii.es/scielo.php?script=sci_arttext\&pi$d=S 0211-57352008000200006$
Valls, Ramón, "El concepto de dignidad humana", Revista de Bioética y Derecho, no.23, diciembre 2011, p.1, Recuperado el 30 de septiembre de 2021 de: https://www.redalyc.org/pdf/783/78339725004.pdf

Varela Pinedo, Luis F., "Salud y calidad de vida en el adulto mayor", Revista Peruana de Medicina Experimental y Salud Pública, vol.33, no.2, abril-junio 2016. Recuperado el 15 de septiembre de 2021 de: http://www.scielo.org.pe/scielo.php?script=sci_arttext\&pid=S1726-46342016000200001

Villarreal, Carla, "El reconocimiento de la capacidad jurídica de las personas con discapacidad mental e intelectual y su incompatibilidad con los efectos jurídicos de la interdicción y la curatela: Lineamientos para la reforma del Código Civil y para la implementación de un sistema de apoyos en el Perú", marzo 2014. Recuperado el 9 de octubre de 2021 de: https://www.corteidh.or.cr/tablas/r36872.pdf. 